\title{
İKİ BORU ARASINDAKİ KATI MADDE TAŞINIMININ HESAPLAMALI AKIŞKANLAR DİNAMİĞİ İLE MODELLENMESİ
}

\author{
(CFD MODELLING OF SEDIMENT TRANSPORT IN ANNULUS) \\ Mehmet SORGUN', Erman ÜLKER²
}

\begin{abstract}
ÖZ
İki boru arasındaki katı madde (sediment) taşınımında basınç farkının yeterli bir doğrulukta belirlenmesi, uygun pompa seçiminde en fazla dikkat edilmesi gereken konulardan birisidir. Katı madde sisteme girdiğinde, basınç farkı artmaktadır. Ayrıca, katı - sıvı çift fazlı sistemde içteki borunun dönmesi akış alanındaki artma nedeniyle basınç farkını azaltmaktadır. Sistemin karmaşıklığı nedeniyle, basınç farkının doğru tahmini tek fazlı akışa göre daha zordur. Bu çalışmada, farklı debi, boru dönme ve delme hızları için geniş kapsamlı bir deneysel çalışma gerçekleştirilmiştir. Deneylerden elde edilen sonuçlar, içteki borunun dönmesinin sediment taşınımını kolaylaştırdığı ve sedimetlerin taşınımı için gerekli olan kritik akış hızını ve basınç gradyanını düşürdüğünü göstermiştir. İki boru arasındaki sediment taşınımı Hesaplamalı Akışkanlar Dinamiği (HAD) kullanılarak modellenmiştir. Diğer parametrelerin yanında basıç farkları da kaydedilmiştir. HAD modelini doğrulamak için basınç farkları deneysel verilerle kıyaslanmıştır. Sonuçlar, HAD modelin basınç farkını yeterli doğrulukta tahmin edebildiğini göstermiştir.
\end{abstract}

Anahtar Kelimeler: Basınç farkı, HAD, İki boru arası akış, Sıvı-katı akışı

\section{ABSTRACT}

Determination of the pressure loss with a sufficient accuracy for sediment transport in annulus is one of the major concerns while proper pump systems selections. When the solid particle is present in the annulus, frictional pressure loss increases. Moreover, rotating inner pipe in solid-liquid system decreases pressure losses due to increase of the free flow area in annulus. Because of the complexity of this system, accurate prediction of frictional pressure loss is more difficult than single flow. The result showed that inner pipe rotation in an annulus ease the sediment transport and decrease pressure gradient and critical velocity that is necessary to initiate sediment movement. In this study, sediment transport in annulus has been modelled using Computational Fluid Dynamics (CFD). Extensive experimental study was conducted at a flow loop for various flow rates, pipe rotation speed, and rate of penetration. Pressure drop within the test section was recorded besides the other test parameters. To verify the CFD model, estimated frictional pressure losses are compared with experimental data. Results show that CFD model is capable of estimating frictional pressure drop. 


\footnotetext{
1 İzmir Katip Çelebi Üniversitesi, Mühendislik-Mimarlık Fakültesi, İnşaat Mühendisliği Bölümü, İzmir, mehmetsorgun@gmail.com (sorumlu yazar)

2 İzmir Katip Çelebi Üniversitesi, Mühendislik-Mimarlık Fakültesi, Makina Mühendisliği Bölümü, İzmir, erman.ulker@ikc.edu.tr
}

\section{GíRiş}

Su ile katı madde (sediment) taşınımına mühendisliğin değişik branşlarındaki pekçok uygulamada rastlanmaktadır. Basınçlı akımlarda katı maddelerin hidrolik taşınımı, en az enerji kullanarak en fazla katı madde miktarının belirli iki nokta arasında taşınımı olarak tanımlanmaktadır [1]. Katı madde taşınımı, borularda ve sondaj kuyularında taşıyıcı olarak akışkan kullanılarak yapılabilmektedir. Katı maddelerin hidrolik taşınımı uygulamaları, madenlerin, kimyasal katı maddelerin ve sondaj esnasında matkabın kuyulardaki formasyonları kesmesi sonucu oluşan kesintilerin (katı madde) etkin bir şekilde yüzeye taşınması olarak sayılabilir. Akışkanın katı madde taşımasını etkileyen pek çok faktör bulunmaktadır. En önemlileri ise, akışkanın hızı ve reolejik özellikleri, katı maddenin özellikleri (dane çapı, özgül ağırlığı), boru çap1, kat1 madde konsantrasyonudur. Sondaj operasyonlarında ise bu parametrelere içteki borunun (tijin) dönme hızı ve kuyu eğimi eklenmektedir.

Katı madde taşınımında dikkat edilmesi gereken en önemli konulardan birisi, sediment yatağının oluşmasını engellemektir. Sedimentlerin taşınması için gerekli olan bir akışkan hızına ihtiyaç bulunmakta olup bu akışkan hızı kritik akışkan hızı olarak ifade edilmektedir. Eğer akışkan hızı kritik akışkan hızından küçükse, sedimentler taşınamamakta ve sediment yatağı oluşmaktadır. Sediment yatağının oluşumu, yük kaybı, borunun tıkanması gibi problemlere yol açmaktadır. Pek çok araştırmacı, kritik akışkan hızının hesaplanabilmesi için deney sonuçlarından yararlanarak ampirik denklemler önermiştir [2-6]. Türetilen denklemler birbirlerinden farklı sonuçlar vermekte olup, bu denklemlerin yalnızca o deney şartları için geçerli olduğu unutulmamalıdır.

Katı madde kalınlığını etkileyen en önemli faktörün kayma gerilmesi olduğu daha önce yapılan çalışmalarda belirtilmiştir [7-8]. Katı madde üzerine etki eden kayma gerilmesinin ise basınç farkı ile ilişkili olduğu kuvvet dengesinden ortaya çıkmaktadır. Bu nedenle katı madde kalınlığını kontrol eden temel faktör basınç farkıdır [9]. Literatürde, basınç farkı ve katı madde kalınlığının hesaplanabilmesi çok sayıda mekanistik model ortaya konmuştur. Modeller, iki ve üç tabakalı modeller olarak ikiye ayrılmaktadır[10-14]. İki tabakalı modeller, durağan sediment yatağı ve akışkan akım alanından oluşmaktadır. Üç tabakalı modellerde ise bu tabakalara ayrıca askıdaki sediment partiküllerinin oluşturduğu tabaka da eklenmektedir.

Son y1llarda bilgisayar teknolojisinin gelişmesi ile hesaplamalı akışkanlar dinamiği (HAD) pek çok mühendislik probleminde kullanılmaya başlanmıştır. Literatürde birçok HAD kullanılarak yapılan çalışma olmasına rağmen, katı madde taşınımının modellenmesi ile ilgili olanların sayısı oldukça azdır. Alizadehdaknel vd. [15] çok fazlı akışlardaki basınç farkını tahmin etmek için bulanık mantık ile HAD modellerinin performanslarını karşılaştırmış ve HAD sonuçlarının bulanık mantıktan daha iyi sonuçlar verdiğini keşfetmiştir. Sun vd. [16] HAD kullanarak içteki borunun dönmesinin sediment taşıması üzerindeki etkisinin simülasyonunu yapmıştır. Sorgun vd [17] HAD ve SVR (Support Vector Regression) kullanarak iki boru arasından geçen Newtonyen ve non-Newtonyen akışkanların basınç 
farklarını tahmin etmişlerdir. Akhshik vd. [18] dinamik çarpma işlemini hesaba katarak kesinti taşınımını bileşik HAD-DEM (ayrık elemanlar methodu) uygulaması ile simülasyonunu sunmuştur. Fu vd. [19] HAD kullanarak yatay eş merkezli borular arasındaki akışkan ve katı madde etkileşimini modellemiştir.

$\mathrm{Bu}$ çalışmanın amacı, iki boru arasındaki sediment taşınımını Hesaplamalı Akışkanlar Dinamiği (HAD) kullanarak modellemek ve sediment taşınımı için kritik bir parametre olan basınç farkını hesaplamaktır.

\section{DENEYSEL ÇALIŞMA}

Bu çalışmada, kuyu içindeki katı madde taşınımını simüle etmek için Şekil 1'de gösterilen deney düzeneği tasarlanmıştır. Deney düzeneği, akış döngüsü $4.6 \mathrm{~m}$ uzunluğundaki yatay $0.076 \mathrm{~m}-0.038 \mathrm{~m}$ iç içe borulardan oluşmaktadır. Katı madde taşınımı iki boru arasında olmaktadır. Dış boru şeffaf ve akrilik olup sediment hareketi ve sediment yatağının oluşumu gözlemlenebilmektedir. İç boru ise çelik olup, içinde akış bulunmamaktadır. İçteki boru 120 (d/d) ya kadar dönebilmektedir.

Sediment taşınımı deneylerinden bir kesit Şekil 2'de gösterilmiştir. Bu çalışmada, sisteme su enjektesi için santrifüjlü pompa kullanılmıştır. Akış hızı manyetik debi metre kullanılarak ölçülmüş ve havalı akım kontrol edici ile kontrol altında tutulmuştur. Deneylerde akışkan olarak su kullanılmıştır. Tablo 1'de sediment taşınımı deneylerinde kullanılan parametrelerin minimum ve maksimum değerleri verilmiştir. Delme hızı, sedimentlerin motor yardımıyla helozonlu vida kullanılarak akış sistemine dahil edilmesi ve sedimentlerin depolandığı tankın ağırlığının değişimi takip edilmek suretiyle ölçülmektedir. Bu çalışmada 90'dan fazla deney yapılmıştır.

Tablo 1. Sediment taşınımı deneylerinde kullanılan parametrelerin minimum ve maksimum değerleri

\begin{tabular}{ccc}
\hline & Minimum & Maksimum \\
\hline Suyun akış hızı (m/s) & 0.64 & 1.3 \\
\hline Boru delme hızı (m/s) & 0.00127 & 0.0038 \\
\hline Boru dönme hızı (d/d) & 0 & 120 \\
\hline $\begin{array}{c}\text { Sediment tane boyutu } \\
(\mathrm{mm})\end{array}$ & 3 & 3 \\
\hline
\end{tabular}

Deneylere başlarken, öncelikle pompa çalıştırılmış, suyun kapalı sistemde devir daim etmesi sağlanmış ve istenilen akış debisi ayarlanmıştır. Sonra sedimetlerin bulunduğu tankın vanası açılmış ve elektrik panosundan boru delme hızı ve içteki borunun dönme hızı ayarlanarak sedimentler sisteme verilmiştir. Sistemin dengeye ulaşması ve sediment yatakların oluşması beklenmiştir. Sistem dengeye ulaştıktan sonra sediment yatağı kalınlığı deney düzeneği üzerindeki 5 farklı kesitte ölçülmüştür. Sediment yatağı kalınlığı, kesinti yatağı alanın kuyu içindeki toplam akış alanına oranıdır ve boyutsuzdur. 
Ayrıca, veri toplayıcı program kullanılarak, akış debisinin yanında akışın tam gelişmiş olduğu bölgede basınç farkı da her saniye için kaydedilmiştir.

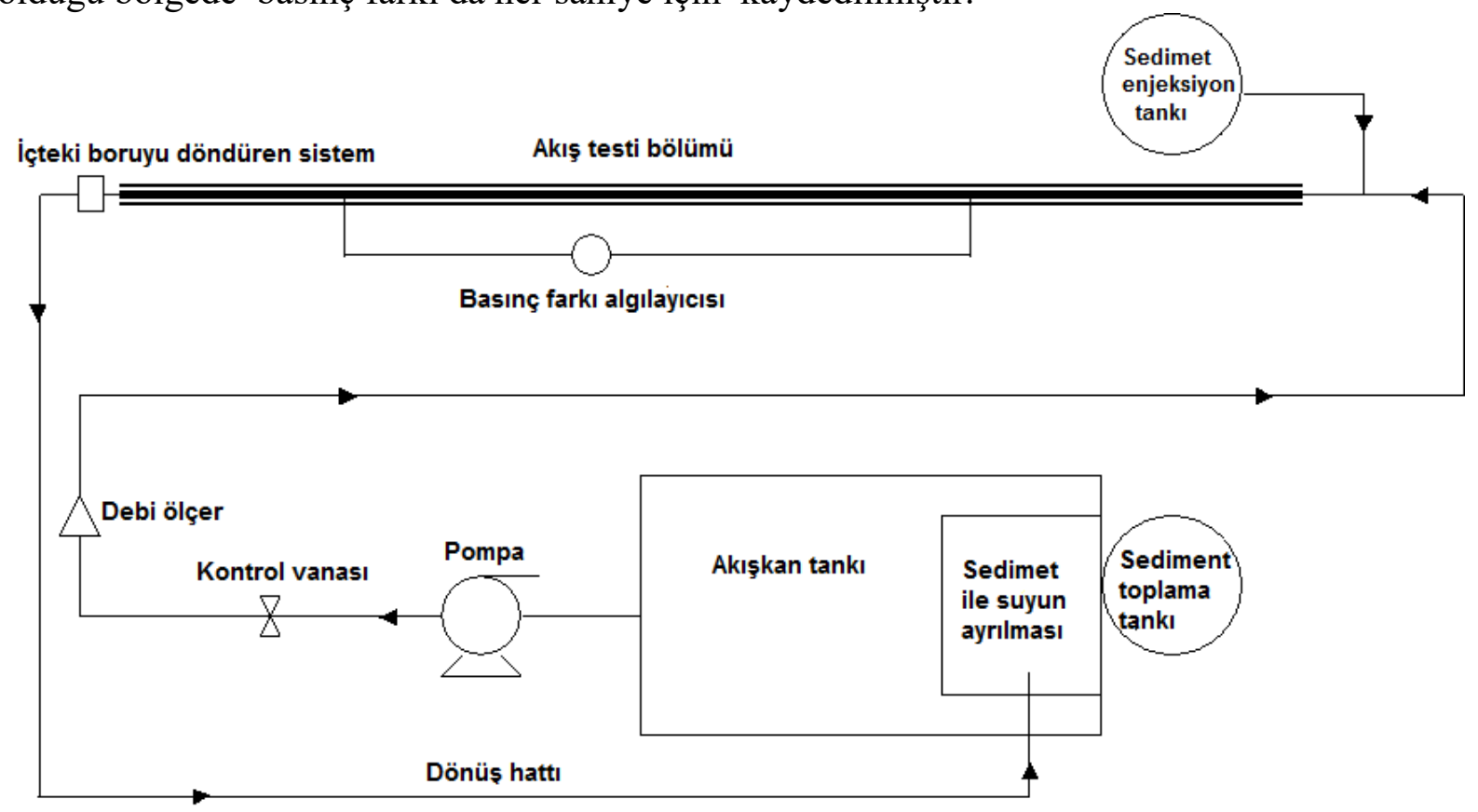

Şekil 1. Deney düzeneğinin şematik gösterimi (Sorgun et al., 2011)

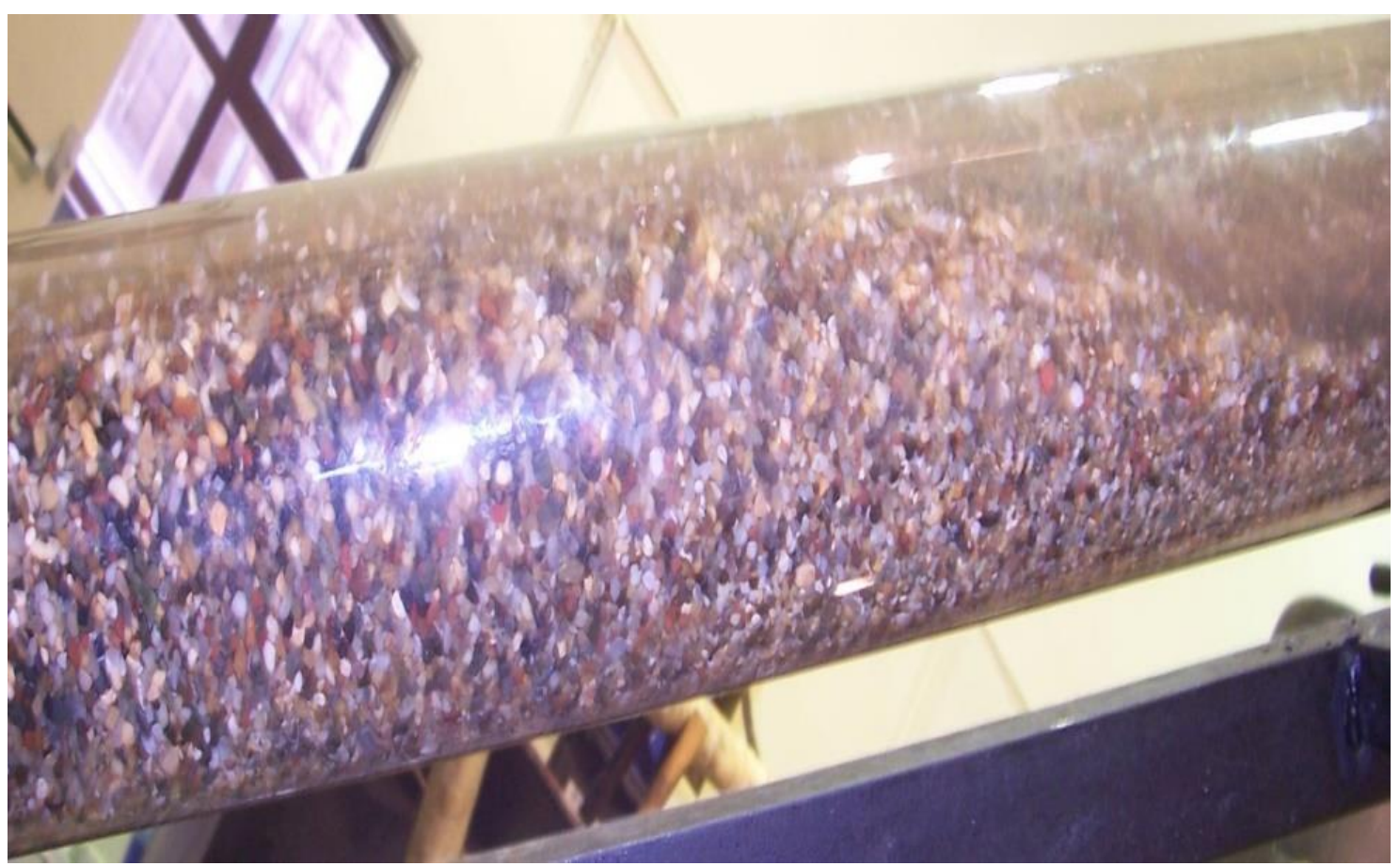

Şekil 2. Sediment taşınımı deneyi sırasında deney düzeneği kesitinden bir görünüş 


\section{HESAPLAMALI AKIŞKANLAR DİNAMİĞİ MODELINIIN ÇÖZÜM METODU}

Bu çalışmada, yatay kuyulardaki sıvı-katı akışın değişik akışkan hızları, delme hızları ve boru dönme hızları için Euler-Euler HAD modeli kullanılarak simülasyonu hazırlanmıştır.

Hem sıvı hemde katı fazlar için süreklilik denklemi şu şekilde yazılabilir [20,21]

$\frac{\partial}{\partial t}\left(\rho_{f} C_{f}\right)+\nabla \cdot\left(\rho_{f} C_{f} U_{f}\right)=0$

$\frac{\partial}{\partial t}\left(\rho_{s} C_{s}\right)+\nabla \cdot\left(\rho_{s} C_{s} U_{s}\right)=0$

aşağıdaki sınırlarla birlikte

$\mathrm{C}_{\mathrm{f}}+\mathrm{C}_{\mathrm{s}}=1$

dir. Burada $f$ ve $s$ alt indisi sırasıyla akışkan ve katı hali, $\mathrm{C}$ ortalama hacim oranını, $\rho$ yoğunluğu, $U$ hız vektörünü ve $t$ zamanı ifade etmektedir.

Her bir faz için momentum denklemi şu şekilde ifade edilebilir [20-22]

$\rho_{f} C_{f}\left[\frac{\partial U_{f}}{\partial t}+U_{f} \cdot \nabla U_{f}\right]=-C_{f} \nabla \mathrm{P}+C_{f} \nabla \cdot \tau_{f}+C_{f} \rho_{f} g-M$

ve katı hal için

$\rho_{\mathrm{s}} \mathrm{C}_{\mathrm{s}}\left[\frac{\partial \mathrm{U}_{\mathrm{s}}}{\partial \mathrm{t}}+\mathrm{U}_{\mathrm{s}} \cdot \nabla \mathrm{U}_{\mathrm{s}}\right]=-\mathrm{C}_{\mathrm{s}} \nabla \mathrm{P}+\mathrm{C}_{\mathrm{s}} \nabla \cdot \tau_{\mathrm{s}}+\mathrm{C}_{\mathrm{s}} \rho_{\mathrm{s}} \mathrm{g}-\nabla \mathrm{P}_{\mathrm{s}}+\mathrm{M}$

dir. Burada $\mathrm{P}$ basıncı, g yerçekim ivme vektörünü, $\tau$ viskoz gerilme tensörünü, Ps katı basıncını, ve $\mathrm{M}$ sürükleme kuvveti $\left(\mathrm{F}_{\mathrm{d}}\right)$ vw kaldırma kuvveti $\left(\mathrm{F}_{\mathrm{l}}\right)$ nin yaptığ arayüzey momentum transferidir.

\section{HESAPLAMALI AKIŞKANLAR DİNAMIĞİ HESAPLAMALARI}

$\mathrm{Bu}$ çalışmada kullanılan simülasyonlar, sonlu elemanlar metodu ile çözüm yapan ANSYS Workbench 12.1 [23] ticari programı kullanılarak hazırlanmıştır. Yapılan simülasyonlar, programın CFX-Solver bileşeni kullanılarak çözülmüştür. İki boru arasından sediment-su akışı gerçekleştirilmiş, $1.5 \times 10^{6}, 3.5 \times 10^{6}, 5 \times 10^{6}$ dört yüzeyli ă̆ sayısı kullanılarak modellemeler yapılmıştır. $3.5 \times 10^{6}$ ile $5 \times 10^{6}$ dört yüzeyli ağ sayısının sonuçlarının birbirine çok yakın olduğu ve daha fazla ăg sayısını artırmanın sonuçları değiştirmediği görülmüştür. $\mathrm{Bu}$ nedenle, problemin çözüm süresi dikkate alınarak, modelemelerde optimum ağ sayısı olarak $3.5 \times 10^{6}$ tetrahedral ağ sayısı kullanılmıştır. Akış steady (daimi akış) olarak çözülmüştür. Tamamen gelişmiş akım elde etmek için farklı boru uzunlukları kullanılarak birçok simülasyon çalışılmıştır. Sonuç olarak, $700 \mathrm{~mm}$ boru uzunluğu tamamen gelişmiş sıvı-katı akımı vermek için yeterli olduğu gözlemlenmiştir. Boru girişindeki sınır koşulu akışkanın hızı, boru çıkışındaki ise atmosferik basınç olarak tanımlanmıştır. Kesinti yoğunluğu $2650 \mathrm{~kg} / \mathrm{m}^{3}$ ve $3 \mathrm{~mm}$ çapındadır. Türbülans modeli olarak 
çeşitli modeller denenmiş, elde edilen sonuçlar benzer olduğundan, daha hızlı simülasyon süresi elde edilen k- $\varepsilon$ modeli kullanılmıştır. Modellemede, core i5 işlemcili 4 GB ram e sahip olan dizüstü bir bilgisayar kullanılmıştır. Her bir simülasyon yaklaşık 3 saat sürmüştür.

\section{SAYISAL SONUÇLAR}

Bu çalışmada, yatay kuyular içindeki sıvı-katı akışı için basınç gradyanı HAD kullanılarak tahmin edilmiştir. Örnek olarak, Şekil 3 ve Şekil 4 de farklı durumlar için HAD tarafından tahmin edilen basınç gradyanı değerleri ile deneysel veriler karşılaştırılmıştır. Şekil 3' de görüldüğü gibi suyun ortalama akış hızı arttıkça basınç gradyanı artmaktadır.

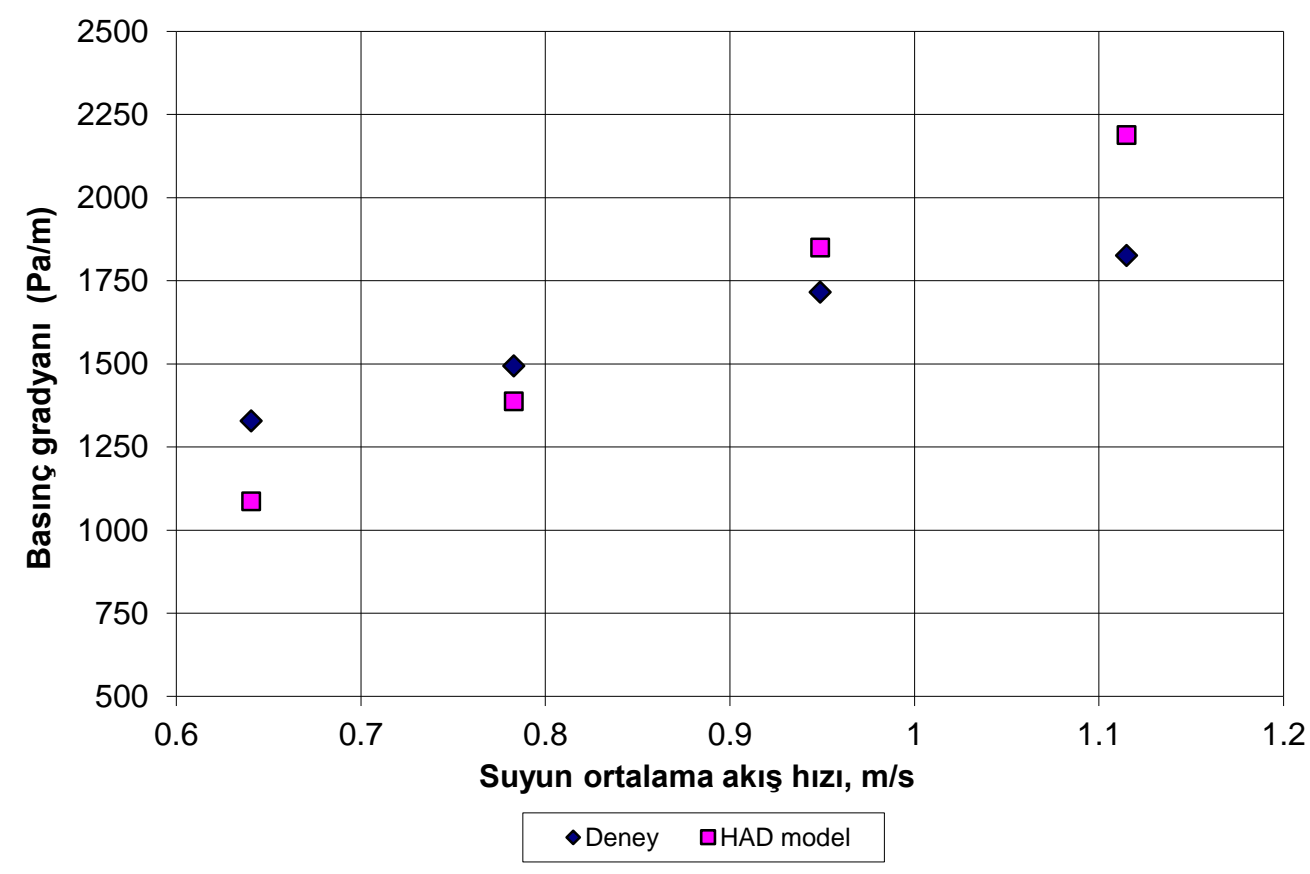

Şekil 3. İç Boru dönme hızı $(\mathrm{d} / \mathrm{d})=0$ ve delme hızının (rop) $=0.00127 \mathrm{~m} / \mathrm{s}$ olduğu durumlar için HAD simülasyonu ile deneysel verilerin karşılaştırılması 


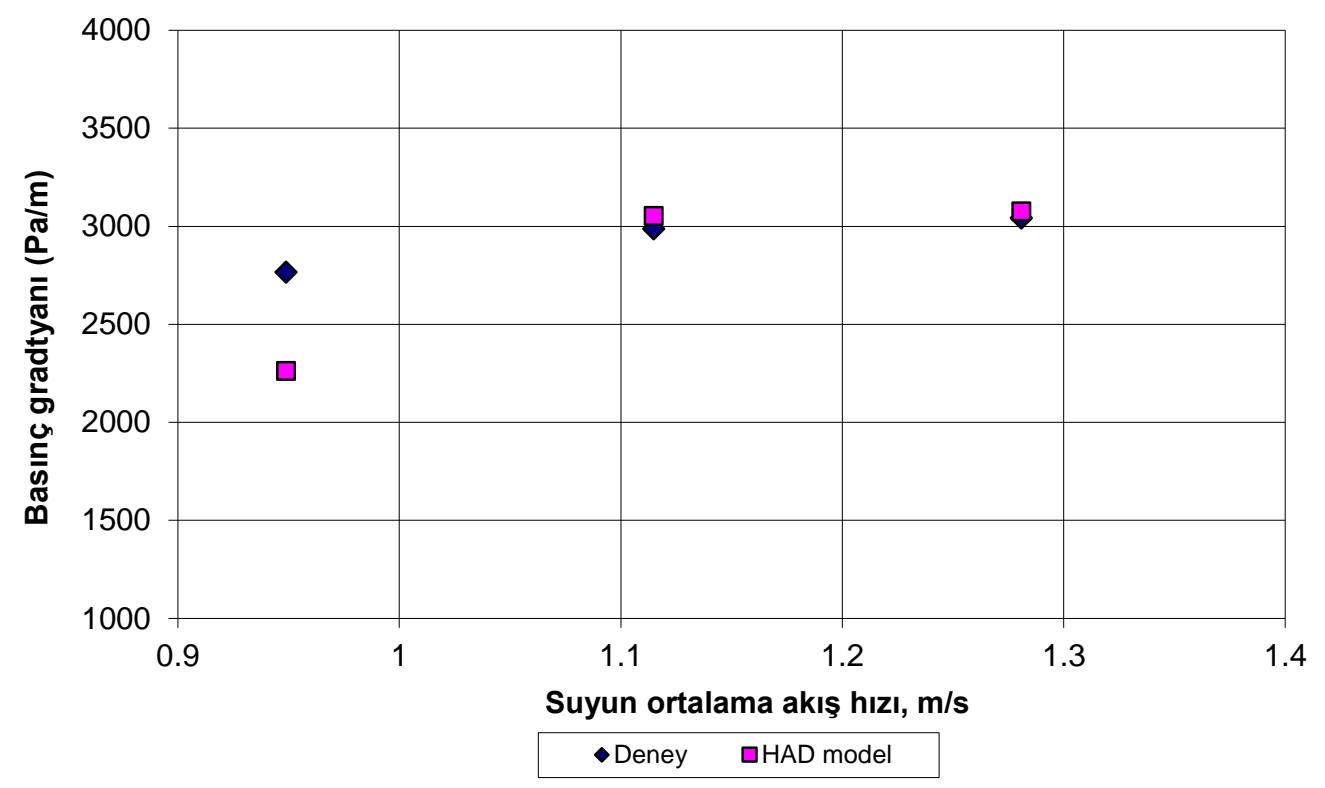

Şekil 4. Boru dönme hızı $(\mathrm{d} / \mathrm{d})=120$ ve delme hızının $($ rop $)=0.0038 \mathrm{~m} / \mathrm{s}$ olduğu durumlar için HAD simülasyonu ile deneysel verilerin karşılaştırılması

HAD'nin doğruluğu ise yapılan tüm simülasyonlar için basınç gradyanının deneysel sonuçları ve model tahminlerini gösteren Şekil 5 incelenerek tespit edilebilir. Şekildeki kesik çizgi $\% \pm 20$ hata payını, düz çizgi ise deneysel veri ile hesaplanan sonuçların tam eşleştiğini göstermektedir. Bu şekillerden de görüldü̆̆̈ gibi, basınç gradyanı çoğu durumda yeterli doğrulukta tahmin edilebilmektedir.

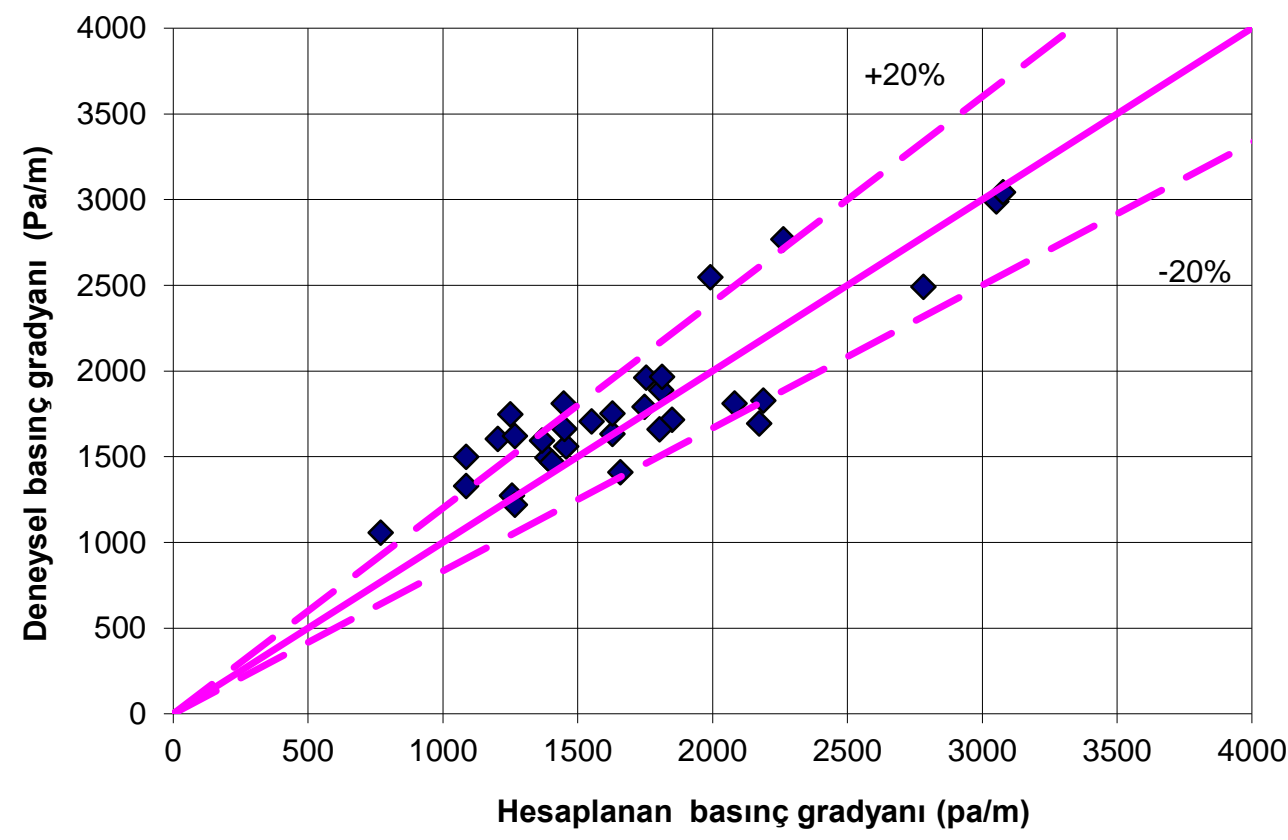

Şekil 5. HAD kullanılarak tahmin edilen basınç gradyanın ölçülen değerlerle karşılaştırılması 
İç boru dönmesinin sediment taşınımına etkisi Şekil 6' da görülmektedir. Şekilden görüldügüü gibi içteki borunun dönmesi sediment taşınımını önemli ölçüde kolaylaştırmaktadır.

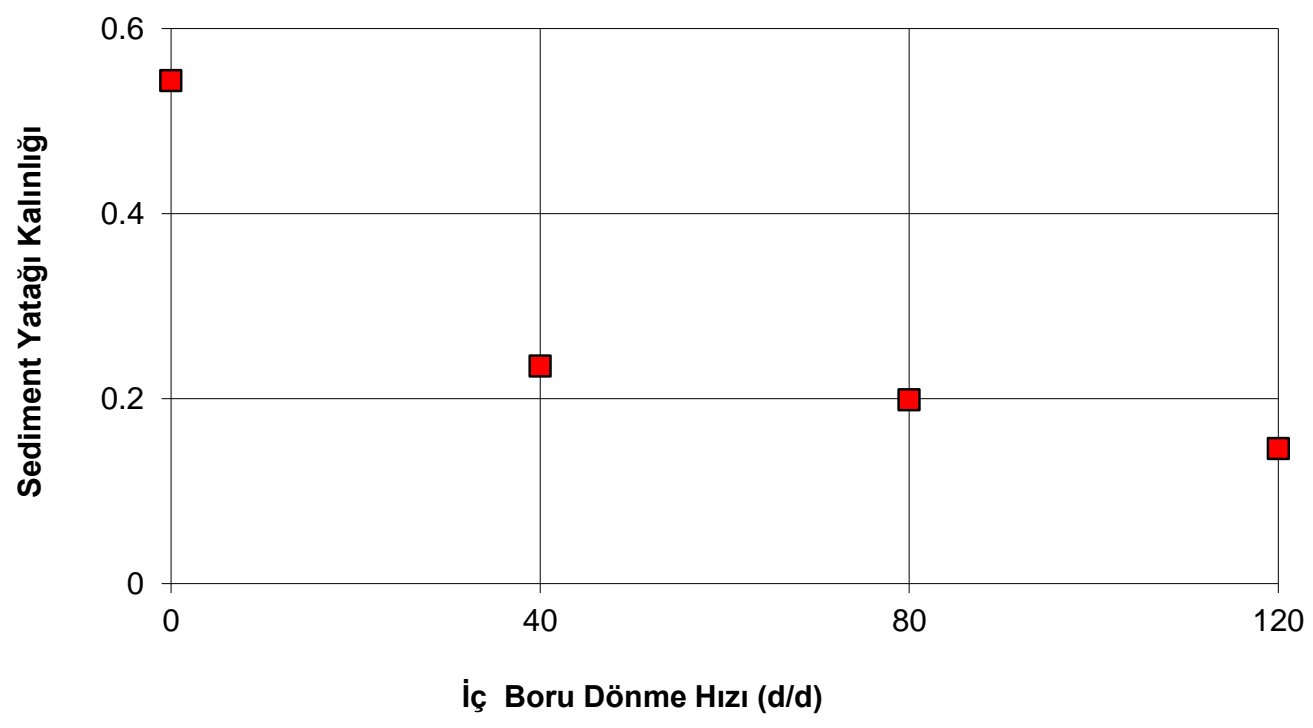

Şekil 6. İç boru dönmesinin sediment taşınımına etkisi

İç boru dönmesinin sediment taşınımı üzerindeki önemli etkilerinden biri kritik akış hızını azaltmasıdır. Şekil 7 de görüldügü gibi iç borunun dönmesi krtik akış hızını önemli ölçüde azaltmaktadır. İçteki boru $40 \mathrm{~d} / \mathrm{d}$ 'da döndürüldüğünde kritik akış hızı $1.28 \mathrm{~m} / \mathrm{s}$ den $0.94 \mathrm{~m} / \mathrm{s}$ ye düşmektedir.

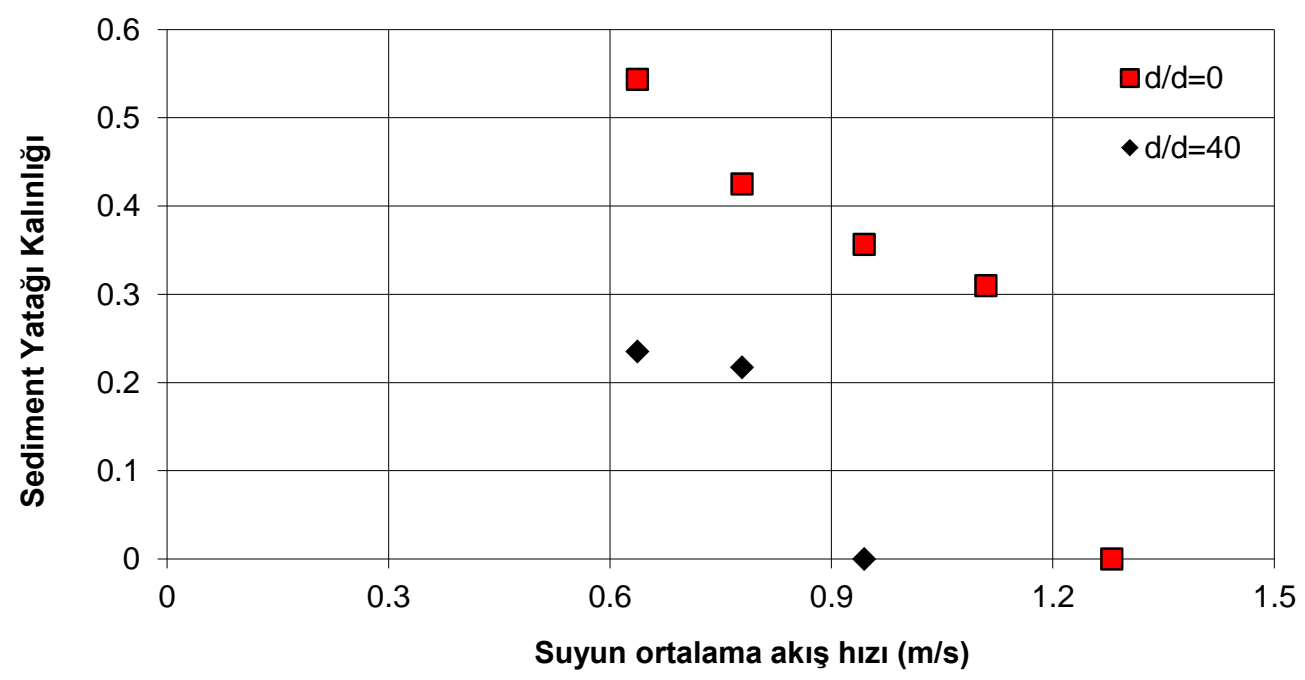

Şekil 7. İç boru dönme hızının sediment taşınımına etkisi 
Şekil 8'de iç boru dönmesinin basınç gradyanına etkisi görülmektedir. Boru döndürüldüğünde sediment yatağı kalınlığındaki azalma nedeniyle basınç gradyanı düşmektedir.

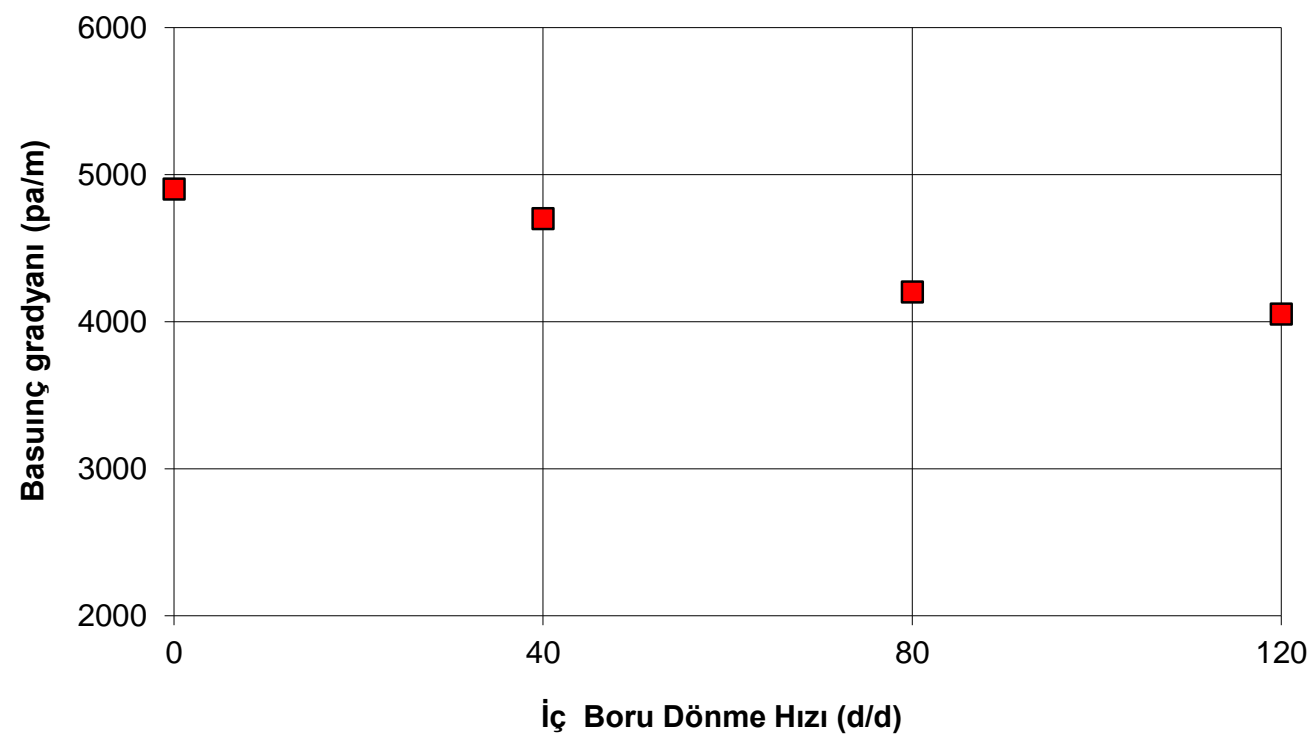

Şekil 8. İç boru dönme hızının basınç gradyanına etkisi

\section{SONUÇLAR}

Bu çalışmada, yatay borular arasındaki sediment taşınımında ortaya çıkan basınç gradyanı HAD yazılımı kullanılarak tahmin edilmiştir. Aşağıdaki sonuçlar elde edilmiştir.

- Model sonuçları, deneylerle karşılaştırıldığında, modelin pek çok durumda basınç gradyanını $\pm 20 \%$ hata payı ile tahmin edebildiği ortaya çıkmıştır.

- Yatay boruların arasından geçen katı-Sıvı iki fazlı akışkanın basınç farkı geçerli bir doğrulukta belirlenebilmesi için HAD kullanılabilinir. Bununla beraber, HAD den elde edilen sonuçlar, boru uzunluğu, kullanılan ağ sayısı gibi pek çok parametreye bağlı olarak değişebilmektedir. Bu nedenle, geliştirilen modeller mutlaka deney sonuçlar ile test edilmeli, doğru sonuç verdiği kanıtlandıktan sonra, farklı simülasyonlar için kullanılmalıdır. Bu kıyaslama ile doğruluğu kontrol edilmeyen modelleri kullanmak hatalara yol açabilir.

- Deneylerden elde edilen sonuçlar, içteki borunun dönmesinin sediment taşınımını kolaylaştırdığ 1 ve sedimetlerin taşınımı için gerekli olan kritik akış hızını ve basınç gradyanını düşürdüğünü göstermiştir. 


\section{KAYNAKLAR}

[1] Acaroğlu E.R., '’Basınçlı Akımlarda Katı Madde Taşınımı', İnşaat Mühendisleri Odası Türkiye İnşaat Mühendisliği, IV. Teknik Kongresi, Rap No: 31, Ankara, 1968.

[2] Kokpinar M.A., Gogus M., ' Critical Flow Velocity In Slurry Transporting Horizontal Pipelines', J. Haydraul.Eng., 2001, 127 (9): 763-771.

[3] ASCE. (1975). Sedimentation engineering, Manuals and reports on engineering practice No. 54, V. A. Vanoni, ed.

[4] Durand R., " Basic relationships of the transportation solids in pipes-Experimental research', Proc. Minnesota Int. Hydr. Conf., 89-103.

[5] Ozbayoglu M.E., Saasen A., Sorgun M., Svanes K., “ Critical Fluid Velocities for Removing Cuttings Bed Inside Horizontal and Deviated Wells", Petroleum Science and Technology, 28: 6, 594-602, 2010.

[6] Zandi, I., and Gavatos, G., ' Heterogeneous flow of solids in pipelines', J. Hydr. Div., ASCE, 93 (3), 145-159.

[7] Kjosnes I., Loklingholm G., Saasen A., Syrstad S.O., Agle A., And Solvang K.A.; "Successful Water Based Drilling Fluid Design for Optimizing Hole Cleaning and Hole Stability", SPE 85330, Presented at SPE/IADC Middle East Drilling Techology Conference and Exhibition, abu Dhabi, UAE, 20-22 October, 2003

[8] Saasen A. And Loklingholm G.; "The Effect of Drilling Fluid Rheological Properties on Hole Cleaning", SPE 74558, Presented at IADC/SPE Drilling Conference, Dallas TX, 2628 February, 2002.

[9] Saasen A., Eriksen N.H., Han Liqun, Labes P. And Marken D., "Is Annular Friction Loss the Key Parameter?", Oil Gas European Magazine, (1)1998, pp.22-24

[10] Doron, P., Granica, D., and Barnea D.," Slurry Flow in a Horizontal Pipes-Experimental and Modeling”, Int.J. Multiphase Flow, 13, 1987, 535-547.

[11] Doron, P. and Barnea D., "'Slurry Flow in Horizontal Pipes- A Three-Layer Model for Solid-Liquid Flow in Horizontal Pipes", Intl. Jour. of Multiphase Flow, 19, 1993, pp. 1029-43.

[12] Larsen, T.I., A.A. Pilehvari And J.J. Azar: "Development of a New Cuttings Transport Model for High-Angle Wellbores Including Horizontal Wells", SPE Drilling\&Completion, Jun. 1997, pp. 129-35.

[13] Nguyen, D. and Rahman S.S.: "A Three-Layer Hydraulic Program for Effective Cuttings Transport and Hole Cleaning in Highly Deviated and Horizontal Wells", SPE Drilling \& Completion, Sep. 1998, pp. 182-9.

[14] Ramadan A., Skalle P., Saasen A.," Application of a three-layer modeling approach for solids transport in horizontal and inclined channels', Chemical Engineering Science, 60 (2005), 2557-2570.

[15] Alizadehdakhel A., Rahimi M., Sanjari J., Alsairafi A.A.,' CFD and Artificial Neural Network Modeling of Two-Phase Flow Pressure Drop', Int Commun Heat Mass Transfer; 36(8):850-6, 2009.

[16] Sun X., Wang K., Yan T., Shao S. ve Jiao J., ''Effect of Drillpipe Rotation on Cuttings Transport Using Computational Fluid Dynamics (CFD) in Complex Structure Wells", Journal of Petroleum Exploration and Production Technology, 4(3), 255-261, 2014.

[17] Sorgun M., Ozbayoglu M.A., Ozbayoglu E.M., 'Support Vector Regression and Computational Fluid Dynamics Modeling of Newtonian and Non-Newtonian Fluids in Annulus With Pipe Rotation'”, J. Energy Resour. Technol. 137(3), 032901 Ekim 21, 2014. 
[18] Akhshik S., Behzad M. ve Rajabi M., 'CFD-DEM Approach to Investigate the Effect of Drill Pipe Rotation on Cuttings Transport Behavior', Journal of Petroleum Science and Engineering, 2015.

[19] Fu T.K., Osgouei R.E. ve Ozbayoglu E.M., 'CFD Simulation of Solids Carrying Capacity of a Newtonian Fluid through Horizontal Eccentric Annular', In Proceedings of the ASME 2013 Fluids Engineering Division Summer Meeting July (pp. 7-11), 2013.

[20] Van Wachem B.G.M., Almstedt A.E.,' Methods for Multiphase Computational Fluid Dynamics', Chemical Engineering Journal 96, 81-98, 2003.

[21] Eesa M. ve Barigou M., 'CFD Investigation of The Pipe Transport Of Coarse Solids in Laminar Power Law Fluids', Chemical Engineering Science, 64(2), 322-333, 2009.

[22] Enwald H., Peirano E., Almstedt A.,' 'Eulerian Two-Phase Flow Theory Applied to Fluidization', International Journal of Multiphase Flow 22, 21-66, 1996.

[23] ANSYS Version 12.1, ANSYS Workbench CFX, ANSYS Inc, 2009.

[24] Sorgun M., Aydin I. ve Ozbayoglu M.E.,' Friction Factors for Hydraulic Calculations Considering Presence of Cuttings and Pipe Rotation in Horizontal/Highly-Inclined Wellbores', Journal of petroleum science and engineering, 78(2), 407-414, 2011.

\section{ÖZGEÇMISS / CV}

Mehmet SORGUN; Doç.Dr. (Assoc.Prof)

Lisans derecesini 2001'de Dokuz Eylül Üniversitesi İnşaat Mühendisliği Bölümü'nden, Yüksek Lisans derecesini 2004'te Celal Bayar Üniversitesi İnşaat Mühendisliği Bölümü'nden, Doktora derecesini 2010 yılında Orta Doğu Teknik Üniversitesi'nden almıştır. Texas A\&M Üniversitesinde 9 ay boyunca misafir araştırmacı olarak bulunmuştur. Sırasıyla 2007 ve 2011 yıllarında Orta Doğu Teknik Üniversitesi tarafından verilen Seçkin Doktora Öğrencisi ve En İyi Doktora Tezi Ödüllerinini almış olup, 2013 yılında ise İzmir Katip Çelebi Üniversitesinde Yayın Onur Ödülüne layık görülmüştür. İzmir Katip Çelebi Üniversitesi İnşaat Mühendisliği Bölümü'nde öğretim üyesi olarak görev yapmaktadır. Temel çalışma alanları: Hesaplamalı Akışkanlar Dinamiği, Hidrolik, Çok fazlı akışlar, Numerik Analiz dir.

He got his bachelors' degree in the Civil Engineering Department at Dokuz Eylul University, Izmir/Turkey in 2001, his master degree in the Civil Engineering Department at Celal Bayar University, Manisal/Turkey in 2004, PhD degree at Middle East Technical University, Ankara/Turkey in 2010. He was a visiting scholar at Texas A\&M University for nine months. He was awarded Distinguished Ph.D. Student in 2007, Best PhD thesis in 2011 at METU and Honor Award in 2013 at Izmir Katip Celebi University. He is still an academic member of the Civil Engineering Department at Izmir Katip Celebi University. His major areas of interests are: Computational Fluid Mechanics, Hydraulics, Multiphase Flow, Numerical Analysis.

\section{Erman ÜLKER; Araş. Gör. (R.A.)}

Lisans derecesini 2005'te Bolu Abant İzzet Baysal Üniversitesi Fizik Bölümü'nden, Yüksek Lisans derecesini 2010 'da The University of Arizona, Uzay ve Uçak Mühendisliği Bölümü'nden, halen Doktora öğrenimini İzmir Katip Çelebi Üniversitesi İnşaat Mühendisliği Bölümü'nde devam ettirmektedir. Aynı zamanda İzmir Katip Çelebi Üniversitesi Makine Mühendisliği Bölümü'nde öğretim elemanı olarak görev yapmaktadır. Temel çalışma alanları: Akışkanlar Mekaniği, Hidrolik, Numerik Analiz üzerinedir.

He got his bachelors' degree in the Physics Department at Abant Izzet Baysal University, Bolu/Turkey in 2005 , his master degree in the Aerospace Engineering Department at University of Artzona, Arizona/USA in 2010, still continue on PhD degree in the Civil Engineering Department at Izmir Katip Celebi University, Izmir/Turkey.. He is still a Research Assistant of the Mechanical Engineering Department at Izmir Katip Celebi University. His major areas of interests are: Fluid Mechanics Hydraulics, Numerical Analysis. 